\title{
Hydrogen-induced electrical and optical switching in Pd capped Pr nanoparticle layers
}

\author{
SHUBHRA KALA and B R MEHTA* \\ Department of Physics, Indian Institute of Technology Delhi, New Delhi 110 016, India
}

\begin{abstract}
In this study, modification in the properties of hydrogen-induced switchable mirror based on $\mathrm{Pr}$ nanoparticle layers is reported. The reversible changes in hydrogen-induced electrical and optical properties of Pd capped Pr nanoparticle layers have been studied as a function of hydrogenation time and compared with the conventional device based on Pd capped Pr thin films. Faster electrical and optical response, higher optical contrast and presence of single absorption edge corresponding to $\mathrm{Pr}$ trihydride state in hydrogen loaded state have been observed in the case of nanoparticle layers. The improvement in the electrical and optical properties have been explained in terms of blue shift in the absorption edge due to quantum confinement effect, larger number of interparticle boundaries, presence of defects, loose adhesion to the substrate and enhanced surface to volume atom ratio at nanodimension.
\end{abstract}

Keywords. Praseodymium nanoparticles; switchable mirror; electrical and optical properties.

\section{Introduction}

Reversible changes in the structural, optical and electrical properties at room temperature on hydrogenation observed in palladium $(\mathrm{Pd})$ capped rare earth hydride $\left(\mathrm{REH}_{x}\right)$ make these materials highly suitable for a number of technologically important applications. Switchable mirrors, display devices and smart windows are some of the potential applications based on such $M-I$ transitions (Huiberts et al 1996; Aruna et al 2006). The $M-I$ transition is observed in both types of rare earth metals: light rare earth La (Greissen et al 1997) and Pr (Mor et al 2001; Kala and Mehta 2007a, b), and heavy rare earth Sm (Kumar et al 2002), Gd (Aruna et al 2004), Dy (Azofeifa and Clark 1997), and Ho (Grier et al 2000). In light rare earth metals, no structural transformation is observed during $M-I$ transition from $\mathrm{REH}_{2}$ to $\mathrm{REH}_{3}$. In heavy rare earth metals, $M-I$ transition is also accompanied by a change in crystal structure. Metal, dihydride and trihydride phases may have different crystal structures. This indicates that the $M-I$ transition is of electronic nature. Band structure and strong correlation models have been proposed to explain the $M-I$ transition (Griessen 2001). Calculations based on Gutzwiller method show $\mathrm{LaH}_{3}$ to be an insulator with an optical bandgap of $1.5 \mathrm{eV}$ or $2.1 \mathrm{eV}$ depending upon whether crystal field effect is included or not (Ng et al 1999). Whereas the experimentally observed values of bandgap are between 2.5 and $3 \mathrm{eV}$. According to strong correlation model, due to availability of one conduction electron per $\mathrm{RE}$ atom in $\mathrm{REH}_{2}$, it possesses the metallic nature.

*Author for correspondence (brmehta@physics.iitd.ernet.in)
During the formation of $\mathrm{REH}_{3}$, additional hydrogen occupies the octahedral position with six nearest RE neighbours and attracts conduction electron to form $\mathrm{H}^{-}$ions. In the $\mathrm{H}^{-}$ion, the two electrons are bonded through different binding energies, the outer electron with $0.7 \mathrm{eV}$ and inner one with $13.6 \mathrm{eV}$. One assumption is that the repulsion between these two on-site electrons results in the narrowing of the $\mathrm{H}$ bands and consequently opening of the bandgap between the rare earth derived metal band and $\mathrm{H}$ band. On the other hand, due to lower binding energy of outer electron, it is engaged in motion between the neighbouring RE atoms and hence unavailable for the conduction. Electronic structure investigations of $\mathrm{LaH}_{x}$ using photoelectron spectroscopy with synchrotron radiation show that La $d$-band near Fermi level diminishes as hydrogen concentration is increased from $x=1 \cdot 9-2 \cdot 9$. Disappearance of La $d$-band near the Fermi level indicates the semiconductor nature of $\mathrm{LaH}_{x}$ at $x=2.9$ (Peterman et al 1981). Optical properties of bulk polycrystalline samples of $\mathrm{ScH}_{2}(3 d), \mathrm{YH}_{2}(4 d)$ and $\mathrm{LuH}_{2}(5 d)$ have been studied by measuring absorptivity as a function of hydrogen concentration in the dihydride state. $\mathrm{YH}_{x}$ shows plasmon absorption characteristic of $\mathrm{YH}_{2}$ metallic state. Due to premature filling of octahedral sites along with the tetrahedral sites by hydrogen, some additional absorption features are also observed at $0.5 \mathrm{eV}$ and $1.25 \mathrm{eV}$ in the $\mathrm{YH}_{2-\varepsilon}$. The position and intensity of absorption features depend mainly on the lattice constant of the dihydride state as well as on hydrogen concentration (Waver et al 1979). Optical properties of RE metals in the thin films as well as in the nanoparticle layers, as a function of hydrogen loading time are not well reported. An understanding of factors that affect the optical properties 
during hydrogen loading and deloading is very important for optimizing and improving device parameters like, optical contrast between dihydride and trihydride states and colour neutrality (constant transmittance between 400 and $800 \mathrm{~nm}$ ) of transparent state. In RE based switchable mirrors, presence of a weak transparency window corresponding to the reflecting dihydride state and a characteristic colour in the transparent trihydride state adversely affects the optical contrast and constant transmittance in the visible region, respectively (Giebels et al 2004). Colour neutrality, high optical and electrical contrasts, and fast reversible switching are key factors from technological point of view. In order to improve constant transmission region, optical contrast and switching kinetics, a number of methodologies have been implemented. To improve colour neutrality, RE metals are alloyed with the $\mathrm{Mg}$ and $\mathrm{MgH}_{2}$ with its large bandgap, acts as a microscopic shutter during hydrogen loading and deloading. Although alloying of $\mathrm{Mg}$ and RE shows significant improvements in colour neutrality, it retards the switching kinetics (van der Sluis et al 1997; Giebels et al 2004). Alloying of $\mathrm{Y}$ and La quenches the transparency window but optical bandgap shifts to lower energy (van Gogh et al 2000). Multilayer structure of $\mathrm{Mg}$ and RE has been utilized to improve switching kinetics while maintaining colour neutrality (van der Sluis et al 1997). In Mg-Y multilayer structures, optical properties and switching kinetics can be controlled by varying the number and thickness of multilayers. Absorption edge of $\mathrm{Y}$ and $\mathrm{Mg}$ multilayer structures having average composition, $\mathrm{Y}_{0.4} \mathrm{Mg}_{0.6} \mathrm{H}_{2 \cdot 4}$, is found to be progressively shifting to lower energy on increasing the multilayer thickness (Giebels et al 2002). Synthesizing material in the nanoparticle form provides a novel methodology of modifying the physical and chemical properties without changing the chemical composition. Blue shift in the absorption edge due to quantum confinement and faster interaction due to the presence of large surface to volume atom ratio at nanodimension can potentially improve the colour neutrality and kinetics, respectively. We have earlier reported the improvement in switching characteristics of $\mathrm{Gd}$ nanoparticle based switchable mirrors (Aruna et al 2004). In this study, electrical and optical properties of switchable mirror based on Pr nanoparticle layers have been investigated and compared with the conventional thin film devices.

\section{Experimental}

Pr nanoparticle layers were deposited by inert gas evaporation technique. Before the deposition of Pr nanoparticle layers, vacuum chamber was evacuated up to a base pressure of $1 \times 10^{-6}$ Torr and then repeatedly purged with argon (Ar) to reduce oxygen partial pressure during deposition. During the deposition of Pr nanoparticles, Ar pressure of $1.2 \times 10^{-3}$ Torr was maintained using the mass flow controller at a flow rate of $13 \mathrm{sccm}$. Subsequently, deposited nanoparticle layers were capped with a thin $\mathrm{Pd}$ layer of $16 \mathrm{~nm}$ thickness without breaking the vacuum at $1 \times 10^{-6}$ Torr pressure. Similarly, Pr thin film sample was deposited at a base pressure of $1 \times 10^{-6}$ Torr and then capped with $16 \mathrm{~nm}$ Pd layer without breaking the vacuum. A pre-calibrated quartz crystal thickness monitor was used to monitor the deposited thicknesses. Pr mass thickness of $90 \mathrm{~nm}$ was kept the same in both the samples. In the remaining part, $\mathrm{Pd}$ capped thin film and nanoparticle samples are mentioned as samples $\mathrm{TF}$ and NP, respectively. Hydrogen loading of the samples, TF and NP, was carried out by introducing hydrogen into the vacuum chamber and maintaining 760 Torr pressure, while during hydrogen deloading, the chamber was evacuated up to $10^{-3}$ Torr pressure with the help of a mechanical rotary pump. Experimental details for in situ electrical measurements are described elsewhere (Kala and Mehta 2007a). For electrical measurements, Keithley 224 programmable current source and Keithley 6517A electrometer resistance meter were used. Structural analysis of $\mathrm{Pd}$ capped nanoparticle layers and thin film samples in as-deposited metallic, hydrogen loaded and deloaded states was carried out by glancing angle X-ray diffractometer (Philips X'pert). UV-vis-NIR double beam spectrophotometer (Perkin-Elmer, Lambda 900) was utilized to record the transmittance spectra in the metallic, hydrogen loaded and deloaded states. Transmission electron microscopic (Philips CM12) studies were carried out to determine surface morphology and particle size.

\section{Results and discussion}

\subsection{Particle size and structural analysis}

Figure 1 shows the TEM micrographs of Pr thin film and nanoparticle layers deposited at $1 \times 10^{-6}$ Torr and $1.2 \times$ $10^{-3}$ Torr pressure, respectively. As these samples are not capped with the protective coating of $\mathrm{Pd}$, the micrographs may correspond to the oxidized samples. Thin film sample shows the compact morphology whereas nanoparticle sample shows particles of diameter, 3-4 $\mathrm{nm}$.

Figure 2 shows the X-ray diffractogram corresponding to the samples TF and NP in the metallic, trihydride and dihydride states. In case of sample TF, XRD peaks are observed at $2 \theta$ and $d$ values of $2 \theta=28.0^{\circ}, d=3.185 \AA$ and $31.7^{\circ}, 2.799 \AA$ corresponding to the (100) and (102) planes of the $d$-hexagonal structure of $\mathrm{Pr}$, respectively. The calculated values of the lattice parameters are $a=3.678 \AA$ and $c=11.795 \AA$. Small deviation from reported values of lattice parameters $a=3.670 \AA$ and $c=11.826 \AA$ for the bulk Pr samples (Hanak and Daane 1961) may be due to the clamping on the substrate and stress during deposition. For the nanoparticle sample, NP, the calculated values of lattice parameters are $a=3.704 \AA$ 

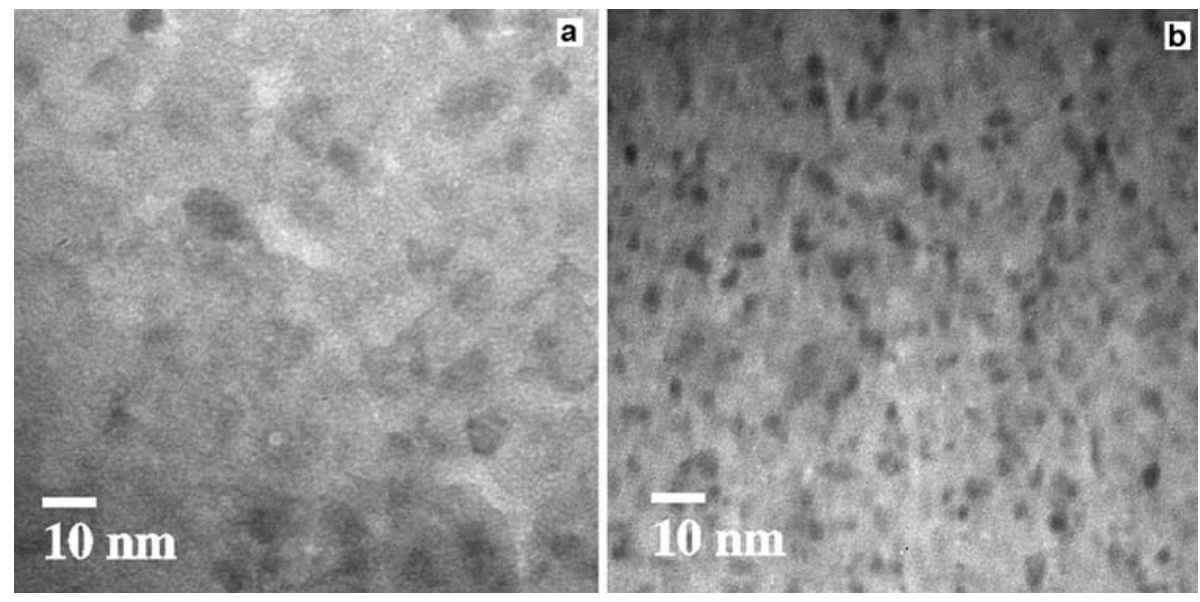

Figure 1. TEM micrographs of the samples (a) thin film and (b) nanoparticle, without Pd overlayer.
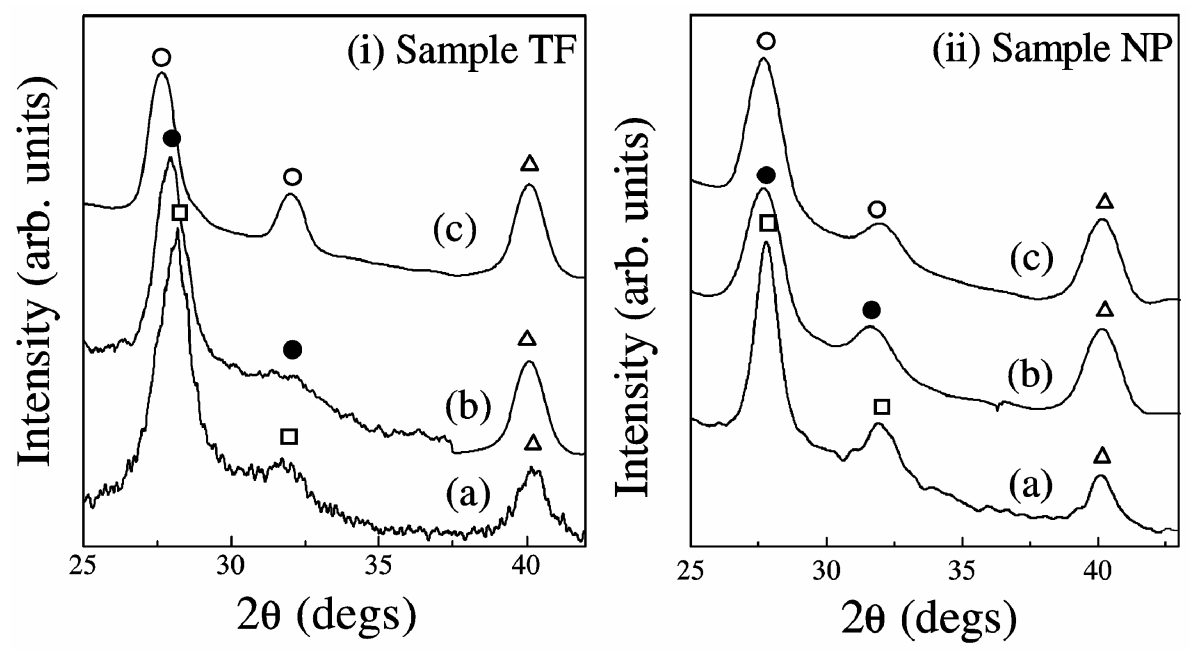

Figure 2. X-ray diffractograms of the samples (i) TF and (ii) NP in (a) metallic, (b) loaded and (c) deloaded states.

and $c=11.823 \AA$. This difference in the lattice parameters of the sample NP with respect to the sample TF may be due to the size induced lattice expansion (Suleiman et al 2003) and presence of large number of stacking faults (Kala and Mehta 2007a). Peak observed at about $2 \theta=40 \cdot 1^{\circ}$ corresponds to Pd (111) plane, which exhibits $f_{c c}$ structure. On hydrogenation, both the samples, NP and $\mathrm{TF}$, undergo structural transformation from $d h c p$ to $f_{c c}$ as reported in the bulk $\operatorname{Pr}$ samples. In hydrogen loaded state, both the samples, $\mathrm{TF}$ and NP, exhibit $f c c$ structure with lattice parameters $5.567 \AA$ and $5.560 \AA$, respectively. On deloading, both the samples, NP and TF, retain their $f c c$ crystal structure with small increase in the lattice parameter. The lattice parameter of TF and NP in the deloaded state is $5.587 \AA$ and $5.586 \AA$, respectively. Similar observation of retaining crystal structure on hydrogen deloading with the increase in the lattice para- meter from 5.483-5.518 $\AA$ has been reported in the bulk Pr samples (Pebler and Wallace 1962).

\subsection{Electrical properties}

Reversible changes in the electrical resistance in the samples, TF and NP, during in situ electrical measurement on hydrogen loading and deloading are shown in figures 3(i) and 3(ii), respectively. At the start of hydrogenation experiment, resistance of the Pr layer from the metal state increases and then saturates, as it transforms to the trihydride state. On subsequent dehydrogenation, the resistance is found to decrease. It may be noted that the resistance of the Pr layer in the deloaded state attains an intermediate value corresponding to the dihydride state. During further cycles of loading and deloading, the 
Table 1. Initial and saturation resistance values in dihydride and trihydride states, respectively in the sample TF and NP. The value of resistance ratio and switching time between dihydride and trihydride state in sample TF and NP are shown.

\begin{tabular}{lcccc}
\hline Sample & $\begin{array}{c}\text { Initial } \\
\text { resistance }(\Omega)\end{array}$ & $\begin{array}{c}\text { Final resistance } \\
(\Omega)\end{array}$ & $\begin{array}{c}\text { Resistance } \\
\text { ratio }\end{array}$ & $\begin{array}{c}\text { Switching } \\
\text { time }(\mathrm{min})\end{array}$ \\
\hline $\mathrm{TF}$ & 74 & 591 & 7.99 & 2.6 \\
$\mathrm{NP}$ & 88 & 899 & 10.21 & 1.56 \\
\hline
\end{tabular}
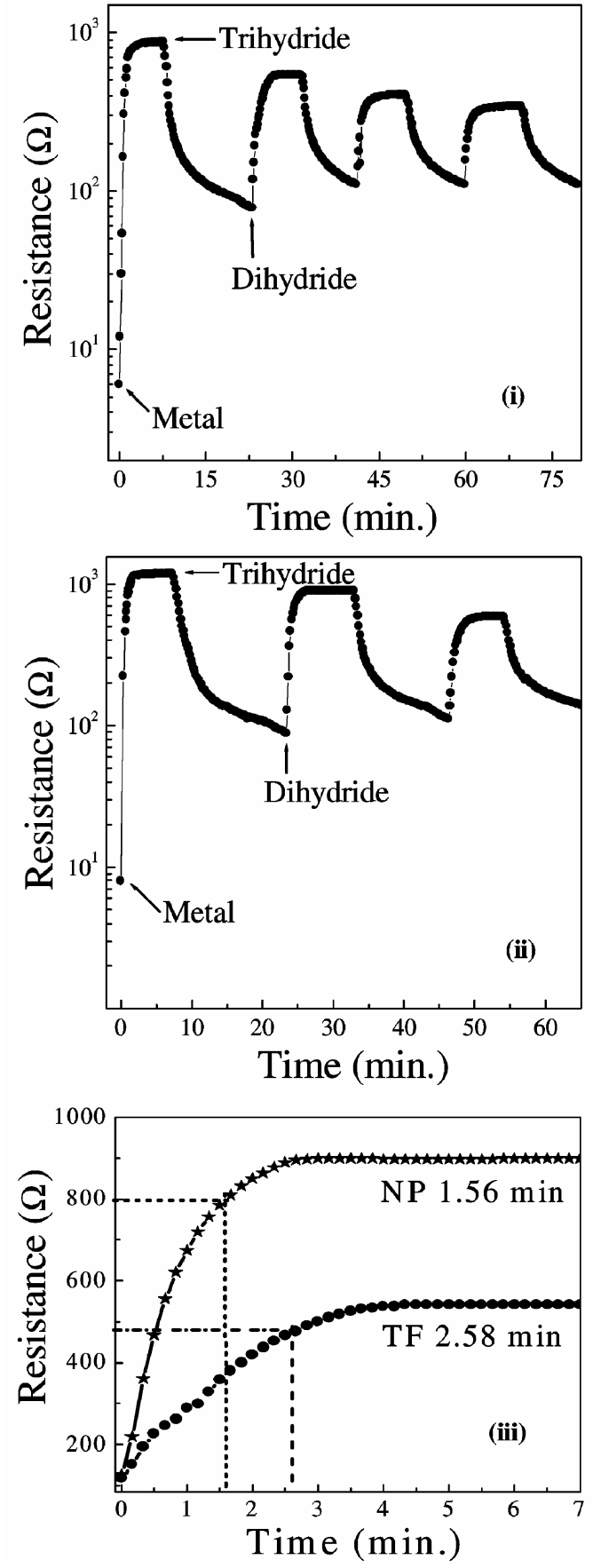

Figure 3. Reversible change in electrical resistance in Pr: (i) $\mathrm{TF}$ and (ii) NP samples. (iii) Switching time in the samples, TF and NP, on exposure to hydrogen ambient. resistance of Pr reversibly changes from the dihydride state to the trihydride state. The gradual decrease in the resistance during reversible cycles of hydrogen loading and deloading in both the samples, TF and NP, indicates the presence of hysteresis. The values of initial resistance in the dihydride state, saturation resistance on hydrogen loading, ratio of the initial and saturation resistance and switching time between dihydride and trihydride states of the samples, TF and NP, are given in table 1 . The mentioned values in the table correspond to the second cycle of hydrogen loading and deloading.

Resistance ratio is observed to increase from 7.99 in $\mathrm{TF}$ to 10.21 in NP. The increase in the resistance ratio between dihydride and trihydride states is due to the larger amount of hydrogen incorporation in nanoparticle samples as nanoparticle layers contain larger number of interparticle grain boundaries and defects (as mentioned earlier in terms of stacking faults). Grain boundaries and defects sites are known as preferred hydrogen active sites in comparison to conventional interstitial sites. Switching time is defined as the time required to reach $90 \%$ of the saturation value. During second loading cycle, sample NP attains $90 \%$ of the saturation value of resistance in $1.56 \mathrm{~min}$ whereas sample TF takes 2.6 min to reach $90 \%$ of the saturation value of the resistance. This indicates that in the sample, NP, electrical switching is faster in comparison to the sample, TF. A detailed study carried out in our laboratory on the improvement in the switching characteristic of Pd capped Pr nanoparticles and thin samples, is presented elsewhere (Kala and Mehta 2007a, b).

\subsection{Optical properties}

Optical properties of Pd capped Pr nanoparticle layers have been investigated by measuring transmittance spectra in as-deposited state and during different hydrogenation time intervals $20 \mathrm{~min}, 30 \mathrm{~min}, 45 \mathrm{~min}$ and $120 \mathrm{~min}$. These transmittance spectra are compared with the corresponding thin film transmittance spectra. Figure 4 shows transmittance spectra of the samples, TF and NP, in the as-deposited state and at different values of hydrogenation time. Low transmittance in as-deposited state in both the samples, TF and NP, indicates the metallic nature of the samples. On hydrogen loading, transmittance of both the samples, TF and NP, increases with respect to the as- 
deposited state. In the sample $\mathrm{TF}$, the transmittance is low up to $30 \mathrm{~min}$ of hydrogen loading time and is observed to increase on 45 min of hydrogen loading time, whereas in the sample NP, significant increase in the transmittance is observed on $20 \mathrm{~min}$ of hydrogen loading time. As mentioned earlier, both the samples are capped with $16 \mathrm{~nm}$ thick Pd layer. In both the samples, the observed lower transmittance on hydrogen loading is due to absorption in $16 \mathrm{~nm}$ thick Pd layer. Absorption coefficient $(\alpha)$ of $\mathrm{Pr}$ layer is calculated by using Lambert's law

$$
T=T_{0} \exp (-\alpha d),
$$

where $T_{0}$ is taken as the transmittance of $\mathrm{Pd}(16 \mathrm{~nm})$ layer deposited on a quartz substrate to eliminate the effect of Pd layer, $T$ is the total transmittance and $d$ the thickness (Lee and Shin 1999). Absorption in the lower energy range 1.5-2 eV has been reported in $\mathrm{ScH}_{2-\varepsilon}, \mathrm{YH}_{2-\varepsilon}$ and $\mathrm{LuH}_{2-\varepsilon}$ due to the plasmon absorption in the metallic dihydride state and premature filling of octahedral sites along with the tetrahedral sites. Thus, the observed
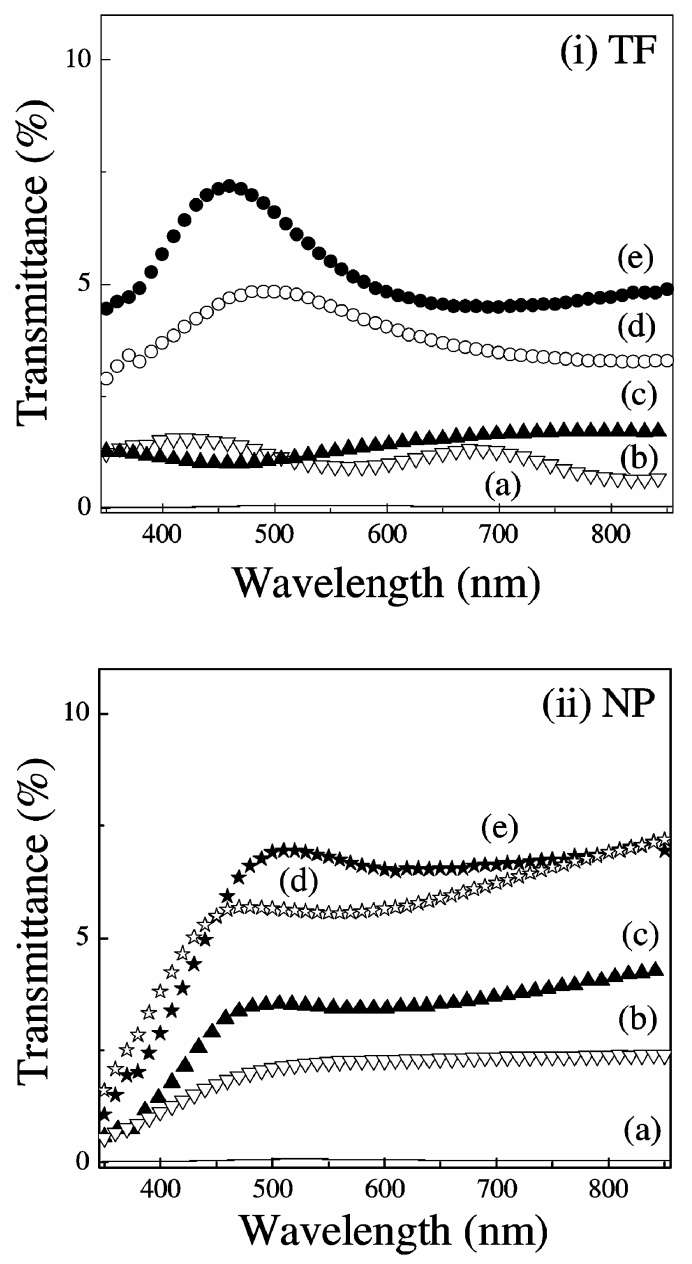

Figure 4. Transmittance spectra of the samples (i) TF and (ii) NP in (a) as-deposited state and after (b) $20 \mathrm{~min}$, (c) $30 \mathrm{~min}$, (d) $45 \mathrm{~min}$ and (e) $120 \mathrm{~min}$ of hydrogenation time. variation in the $\alpha$ value at $1.5 \mathrm{eV}$ can be related to the $\%$ change of Pr into $\mathrm{PrH}_{2+\varepsilon}$ state. The rare earth metals are known to have absorption edge at $2 \cdot 5-3 \mathrm{eV}$ in the trihydride state. Thus, the value of $\alpha$ at $3 \mathrm{eV}$ can be related to the transparent $\mathrm{PrH}_{3-\delta}$ trihydride state. The values of $\alpha$ at $1.5 \mathrm{eV}$ and $3 \mathrm{eV}$ as a function of hydrogenation time are plotted in figure 5. During the complete hydrogenation time of $120 \mathrm{~min}$, value of $\alpha$ at $1.5 \mathrm{eV}$ increases in the sample TF. This indicates that in the sample TF, even after 120 min of hydrogen loading most of the crystallites
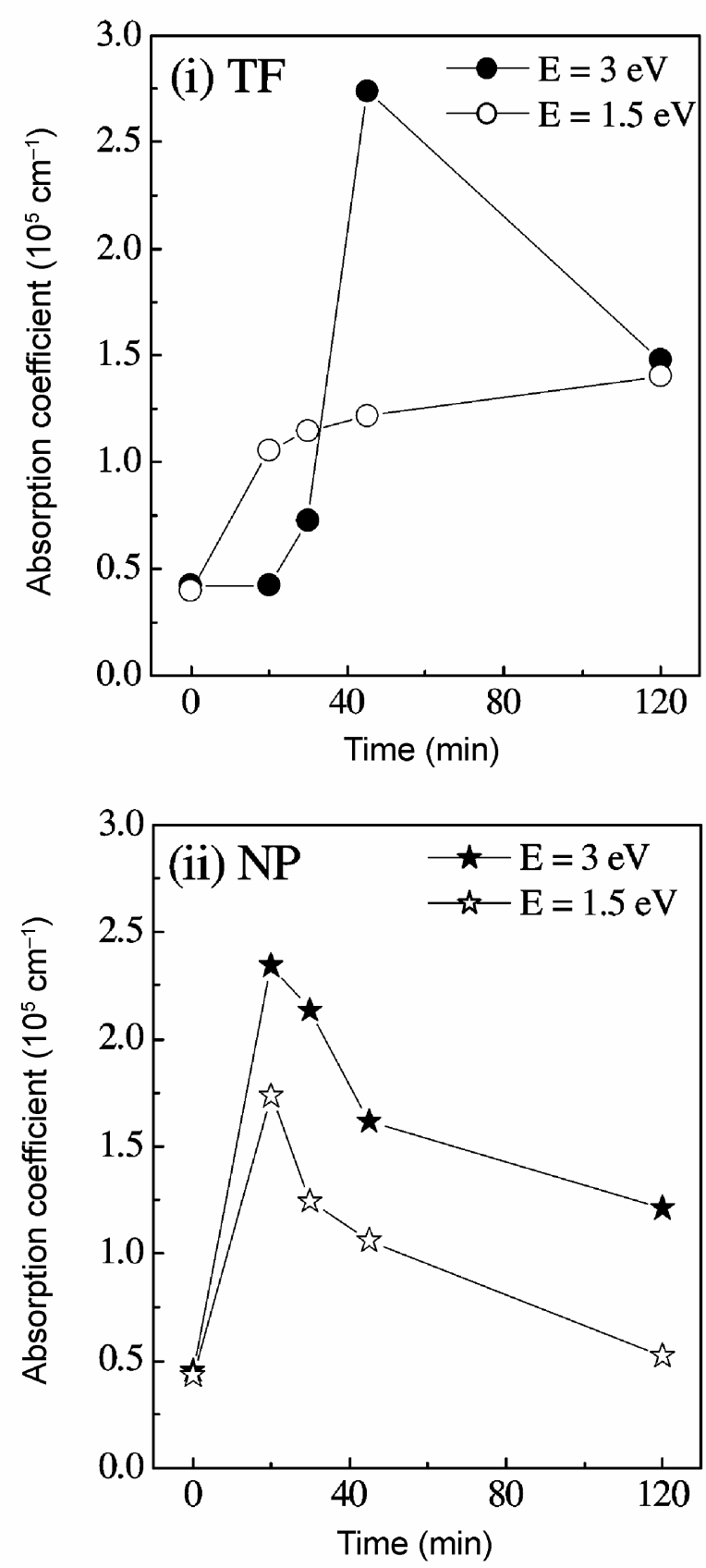

Figure 5. The values of absorption coefficient at 1.5 and $3 \mathrm{eV}$ in the samples (i) TF and (ii) NP as a function of hydrogenation time. 
are still in the dihydride state and are not able to completely transform into trihydride state. The increase in $\alpha$ is due to the increased amount of $\mathrm{H}$ getting incorporated, which results in the conversion of $\mathrm{Pr}$ metal to dihydride state. In the case of sample NP, $\alpha$ at $1.5 \mathrm{eV}$ starts decreasing after $20 \mathrm{~min}$ of hydrogen loading time. This indicates that after 20 min of hydrogen loading the crystallites having dihydride state start getting converted to trihydride state. In the sample NP, the value of $\alpha$ at $3 \mathrm{eV}$ is comparatively higher even on $20 \mathrm{~min}$ of hydrogen loading, whereas in the sample $\mathrm{TF}$, a significant increase in $\alpha$ at $3 \mathrm{eV}$ value is observed only after $45 \mathrm{~min}$ of hydrogen loading, indicating the slow formation of $\mathrm{PrH}_{3-\delta}$ state. At higher loading times the value of $\alpha$ at $3 \mathrm{eV}$ in both the samples decreases. This may be due to the increase in the absorption edge of the trihydride state with increase in hydrogen concentration. Absorption edge of the trihydride state in the samples, TF and NP, after 120 min of hydrogen loading is calculated by Tauc's plot as shown in figure 6 . A detailed explanation of the relationship between the optical, electrical and structural changes in Pr thin film and nanoparticle layers during hydrogenation and dehydrogenation is presented elsewhere (Kala and Mehta 2007b).

The Tauc's plot of the sample, TF, shows absorption edge at $2.59 \mathrm{eV}$ corresponding to the trihydride state along with the weak absorption features in the energy range 1.5-2 eV which indicates the presence of the dihydride state. Whereas in the case of nanoparticle sample NP, no absorption features are observed in the lower energy range. The absorption edge is observed at $2.91 \mathrm{eV}$. This indicates that after $120 \mathrm{~min}$ of hydrogen loading nanoparticle samples are able to transform in the trihydride state whereas sample TF still shows signature of the dihydride state. Absorption edge corresponding to trihydride state is observed to shift from $2.59 \mathrm{eV}$ in the sample

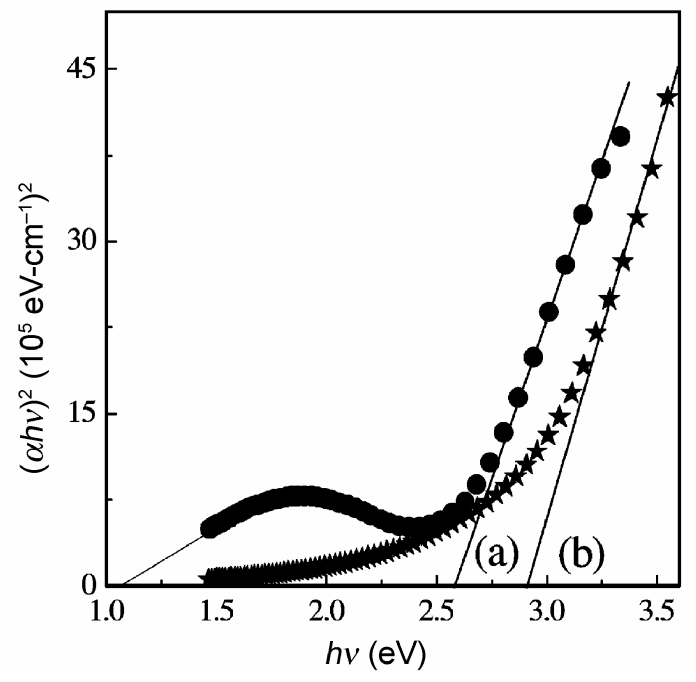

Figure 6. Tauc's plots of the samples (a) TF and (b) NP in the hydrogen loaded state
TF to $2.91 \mathrm{eV}$ in the sample NP. The observed blue shift in the absorption edge on reducing the size of the nanoparticles is due to the quantum confinement effect and larger amount of hydrogen incorporation.

After $120 \mathrm{~min}$ of hydrogen loading, both the samples TF and NP are deloaded. The values of $\alpha$ in the loaded and deloaded states are plotted in the energy range 1.5$3.5 \mathrm{eV}$ as shown in figure 7(i). On deloading, at both the energy values of $1.5 \mathrm{eV}$ and $3 \mathrm{eV}$, the sample NP shows larger change in the value of $\alpha$ in comparison to the sample TF. This shows an efficient conversion of trihydride state back to the dihydride state in the case of sample NP as compared to the sample TF. Optical contrast (OC) provides information about the amount of desorbed hydrogen between dihydride and trihydride states. OC is defined as $\left(T_{\mathrm{T}}-T_{\mathrm{D}}\right) / T_{\mathrm{T}}$, where $T_{\mathrm{T}}$ and $T_{\mathrm{D}}$ are the transmittance in the trihydride and dihydride states, respectively. Higher OC in the sample NP $\sim 50 \%$ is observed in comparison to the sample TF as shown in figure 7(ii). This indicates that in
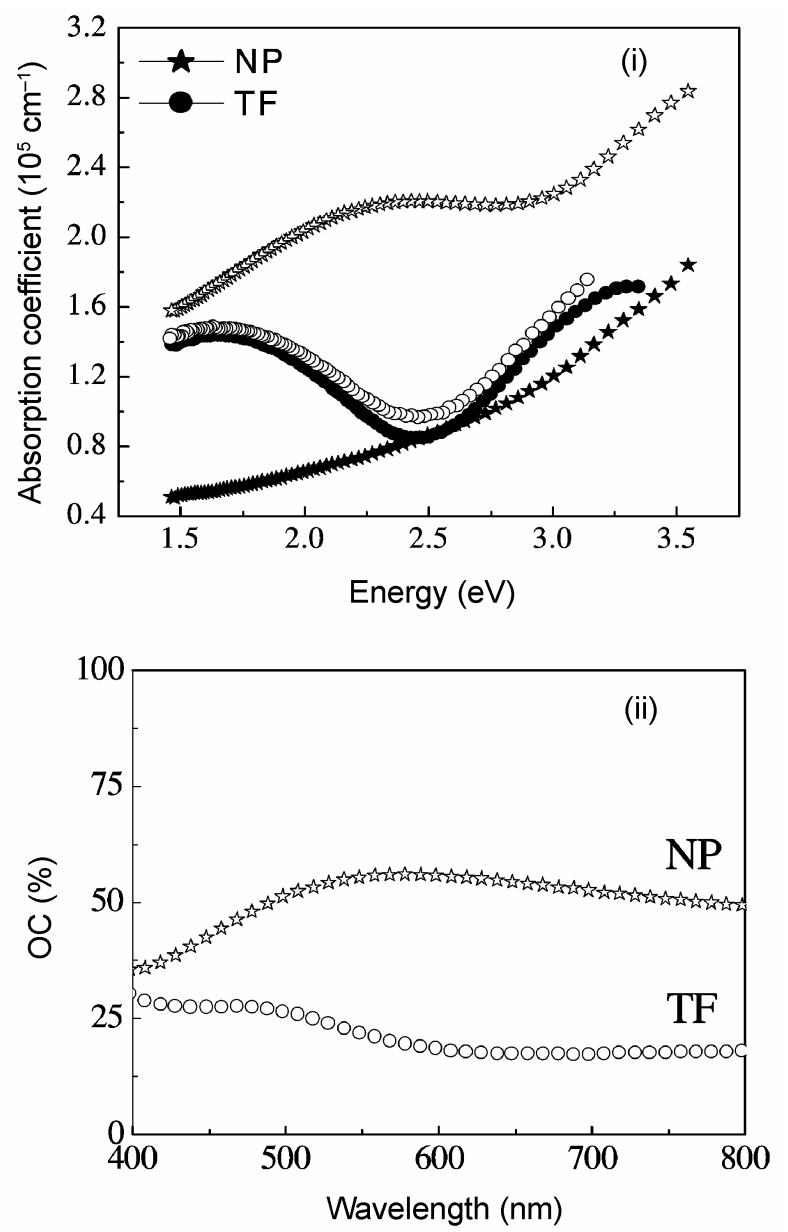

Figure 7. (i) Absorption coefficient spectra in hydrogen loaded (solid symbol) and deloaded (hollow symbol) states for samples TF and NP and (ii) optical contrast between hydrogen loaded and deloaded states in TF and NP samples. 
the case of nanoparticle sample, more hydrogen desorbs during deloading in comparison to the thin film sample.

In both the samples, TF and NP, the electrical switching is faster in comparison to the optical switching. For observing electrical changes, conversion of a smaller $\%$ of metal crystallites to dihydride state and then to trihydride state is sufficient. Whereas, measurable changes in the optical properties can only be observed when a majority of metal crystallites are collectively converted into dihydride state and then into trihydride state. This aspect has been explained in detail elsewhere (Kala and Mehta 2007b).

As mentioned earlier, nanoparticle layers contain large number of grain boundaries and stacking faults in comparison to thin film samples. Grain boundaries and defects are known to be lower energy sites for hydrogen absorption in comparison to the conventional interstitial sites (Pundt 2004). Faster hydrogen absorption and desorption are known to occur through these sites. In the case of sample TF, compact topography and good adhesion to the substrate results in crystallites interlocking and substrate clamping, respectively, during hydrogen loading and deloading. On the other hand, nanoparticle layers have comparatively a loose inter-particle structure, weak adhesion to the substrate due to low ad-atom mobility during inert gas evaporation (Maissel and Glang 1970) and the larger concentration of hydrogen active sites, which results in faster hydrogen absorption/desorption. This explains the faster electrical and optical switching, existence of the single absorption edge corresponding to trihydride state, larger amount of hydrogen absorption/ desorption during loading/deloading and higher optical contrast in the sample NP in comparison to the sample TF.

\section{Conclusions}

A summary of the detailed work carried out on switchable mirror devices based on Pr nanoparticle layers is presented here. Our results show that an all-round improvement in the switching characteristics, electrical and optical contrasts and switching time can be realized by using rare earth nanoparticle layers.

\section{Acknowledgements}

One of the authors (SK) acknowledges the Council of Scientific and Industrial Research, India, for providing a Senior Research Fellowship. This work was supported by
Nano Science and Technology Initiative of the Department of Science and Technology, Government of India.

\section{References}

Aruna I, Mehta B R, Malhotra L K and Shivaprasad S M 2004 Adv. Mater. 16169

Aruna I, Malhotra L K and Mehta B R 2006 Handbook on the phys. and chem. of rare earths (eds) $\mathrm{K}$ A Gschneidner et al (The Netherlands: North-Holland) 36 pp 83, 228

Azofeifa D E and Clark N 1997 J. Alloys Compd. 30532

Giebels I A M E, Isidorsson J, Kooij E S, Remhof A, Koeman N J, Rector J H, van Gogh A T M and Griessen R $2002 \mathrm{~J}$. Alloys Compd. 330-332 875

Giebels I A M E, Isidorsson J and Griessen R 2004 Phys. Rev. B69 205111

Griessen R 2001 Europhys. News 32

Griessen R, Huiberts J N, Kremers M, van Gogh A T M, Kooman N J, Dekker J P and Notten P H L 1997 J. Alloys Compd. 253-254 44

Grier E J, Kolosov O, Petford-Long A K, Ward R C C, Wells M R and Hjorvarsson B 2000 J. Phys. D: Appl. Phys. 33 894

Hanak J J and Daane A H 1961 J. Less Common Met. 3110

Huiberts J N, Griessen R, Rector J H, Wijngaarden R J, Dekker J P, de Groot D G and Koeman N J 1996 Nature 380231

Kala S and Mehta B R 2007a J. Alloys Compd. 43110

Kala S and Mehta B R 2007b J. Appl. Phys. 101124312

Kumar P, Philips R, Mor G K and Malhotra L K 2002 Jpn. J. Appl. Phys. 416023

Lee M W and Shin W P 1999 J. Appl. Phys. 866798

Maissel L I and Glang R 1970 Handbook of thin film technology (New York: McGraw-Hill Book Company)

Mor G K, Malhotra L K and Bhattacharyya D $2001 \mathrm{~J}$. Appl. Phys. 901795

Ng K K, Zhang F C, Anisimov V I and Rice T M 1999 Phys. Rev. B59 5398

Pebler A and Wallace W E 1962 J. Phys. Chem. 66148

Peterman D J, Waver J H and Peterson D T 1981 Phys. Rev. B3 3903

Pundt A 2004 Adv. Eng. Mater. 611

Suleiman M, Jisrawi N M, Dankert O, Reetz M T, Bahtz C, Kirchheim R and Pundt A 2003 J. Alloys Compd. 356-357 644

van der Sluis P, Ouwerkerk M and Duine P A 1997 Appl. Phys. Lett. 703356

van Gogh A T M, Nagengast D G, Kooij E S, Koeman N J and Griessen R 2000 Phys. Rev. Lett. 852156

van der Sluis P, Ouwerkerk M and Duine P A 1997 Appl. Phys. Lett. 731826

Waver J H, Rosei R and Peterson D T 1979 Phys. Rev. B19 4855 